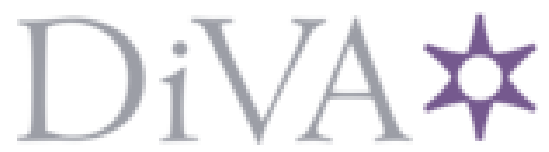

http://www.diva-portal.org

This is the published version of a paper published in Wireless networks.

Citation for the original published paper (version of record):

Masoudi, M., Zaefarani, H., Mohammadi, A., Cavdar, C. (2017)

Energy efficient resource allocation in two-tier OFDMA networks with QoS guarantees.

Wireless networks

https://doi.org/10.1007/s11276-016-1442-5

Access to the published version may require subscription.

N.B. When citing this work, cite the original published paper.

Permanent link to this version:

http://urn.kb.se/resolve?urn=urn:nbn:se:kth:diva-205192 


\section{Energy efficient resource allocation in two-tier OFDMA networks with QoS guarantees}

Article in Wireless Networks · January 2017

DOI: $10.1007 / \mathrm{s} 11276-016-1442-5$

\section{CITATIONS}

0

4 authors:
Meysam Masoudi

KTH Royal Institute of Technology

4 PUBLICATIONS 1 CITATION

SEE PROFILE

\section{Abbas Mohammadi}

Amirkabir University of Technology 215 PUBLICATIONS 3,661 CITATIONS

SEE PROFILE
READS

42
Hamid reza Zaefarani

Amirkabir University of Technology

3 PUBLICATIONS 0 CITATIONS

SEE PROFILE

\section{Cicek Cavdar}

KTH Royal Institute of Technology

61 PUBLICATIONS 374 CITATIONS

SEE PROFILE

Some of the authors of this publication are also working on these related projects: 


\title{
Energy Efficient Resource Allocation in Two-Tier OFDMA Networks with QoS Guarantees
}

\author{
Meysam Masoudi · Hamidreza Zaefarani · \\ Abbas Mohammadi . Cicek Cavdar
}

the date of receipt and acceptance should be inserted later

\begin{abstract}
In this paper, we study joint power and subchannel allocation problem for OFDMA based femtocell networks with focus on uplink direction. We minimize the aggregate power of all Femto user equipments and maximize the total system energy efficiency while satisfying the minimum required rate of all users. An interference limit constraint is considered to protect the QoS of macrocells. The original problem is a mixed-integer non-convex optimization problem which is converted to a convex problem using the time-sharing concept. Three algorithms are proposed to provide a scheme to optimize the goal function while meeting the constraints. The complexity order of all algorithms was investigated and was compared to other alternative solutions. The analytic and simulation results have demonstrated that the proposed algorithms could achieve significant power saving and better energy efficiency compared to existing algorithms.
\end{abstract}

Keywords Femtocell - Resource Allocation - Power Minimizations · Energy Efficiency · Convex Optimization

\section{Introduction}

Growing demand for high quality services in wireless cellular networks is one of the most challenging issues to be met in near future. Furthermore, the study in [1] shows that indoor usage comprises approximately $50 \%$ of all voice calls and about $70 \%$ of data traffic. Consequently, operators are willing to provide high data rate services to the indoor users. This characteristic is vital

M. Masoudi, H.R. Zaefarani, A. Mohammadi

Microwave and Wireless Communication Research Laboratory,

Electrical Engineering Department, Amirkabir University of Technology (Tehran Polytech-

nic), Iran E-mail: meysammasoudi@aut.ac.ir; h-r-z@aut.ac.ir ; abm125@aut.ac.ir

C. Cavdar

Wireless@KTH, KTH Royal Institute of Technology, Sweden E-mail: Cavdar@kth.se 
in next generation networks such as LTE-A and probably 5G [2]. A promising solution to meet these requirements is to employ small cells overlaying a macrocell [3]. In particular, femtocells as a heterogeneous network solution, have received much more attention than other solutions from both academia and industry [4]. Femtocell base stations (FBSs) are low power, short range and low cost access points which are deployed by the end users. FBSs utilize the infrastructures such as DSL, Cable Modem or separate RF as a backhaul to connect to the operator primary network [5]. In addition, femtocells can boost network performance in terms of high data rate services due to the close proximity of femtocell user equipment (FUEs) to their indoor installed FBS [6], [7]. Although femtocells are beneficial to both operators and users, many technical challenges associated with femtocell deployment arise such as high interference level in the network, radio resource utilization, fairness and complexity [8]. Moreover, energy saving methods attract the attention of researchers for next generation wireless networks such as 4G and 5G [9].

\subsection{Literature Review}

The resource allocation problem in orthogonal frequency-division multiple access (OFDMA) cellular networks has been studied in the literature to deal with the challenges and to enhance the network performance. Subchannel allocation and power control are promising ways of dealing with an interference problem. Especially co-channel spectrum allocation is a preferable spectrum utilization from the industry standpoint due to its efficient spectrum utilization [10]. However, the subchannel assignment and power allocation are complicated optimization problems [11]. Challenges of radio resource management for femtocell networks are discussed in [4]. In [12] the authors proposed a scheme to reduce the downlink interference while maintaining the QoS of users. The authors in $[13]$ proposed a distributed energy efficient resource management algorithm using game theory to mitigate the excessive interference to achieve a better throughput. Also in [14], a joint subchannel and power allocation algorithm for the downlink of an OFDMA network deployment is proposed to maximize the sum rate of all FUEs while guaranteeing macrocell user equipment (MUEs) to reach their minimum QoS requirement. A novel spectrum partitioning scheme using directional antennas is proposed in [15] to reduce the interference.

To address the power allocation strategies, in [16] the authors proposed a game theoretic approach for power allocation. The performance is evaluated based on average femtocell and total throughput. Energy efficient power allocation models are also proposed in [17] and performance of the femtocell network is analyzed in terms of the blocking probabilities with partially open channels. In [18], the authors proposed an algorithm to maximize the minimum data rate of FUEs while MUEs must reach their required rate. Heuristic approaches are adopted in [19] and [20]. In [19] a multiobjective approach using genetic algorithm is adopted. The authors in $\underline{20]}$ proposed a generalized 
particle swarm optimization model for adaptive resource allocation for single layer and cross layer optimizations. Power control strategies to manage minimum outage probability for femtocells are considered in [21]. In [22], based on the Lagrangian dual decomposition method, the authors derived a suboptimal resource allocation policy for femtocells in uplink direction. The authors in $\underline{[23]}$ derived a closed-form relationship between two conflicting parameters, spectral efficiency and energy efficiency.

Energy efficient resource allocation guarantees that maximum throughput is achieved with minimum energy consumption. The studies in [9], [24-27] analyzed their system from energy efficiency perspective. In [9], the authors proposed two energy efficient algorithms but they do not consider neither guaranteeing the QoS of macrocell users nor the channel allocation. Also they consider only downlink power control. However, we focused on uplink and channel allocation as well as more constraints is considered. In [24] instead of energy efficiency, the total revenue of a system is maximized. They considered cognitive network. Also their focus was only on power control and there is no channel allocation. So it is not fair to compare our results with theirs. In [25] a non-cooperative game is used to model the power control and numerical search methods are adopted to solve the problem. In this study the user rate guarantee was not taken into account as in our case. Moreover, the system model has only one tier (say only macrocell). In [26] a multi-objective approach is considered towards maximizing the individual energy efficiency rather than total energy efficiency, focusing on single-cell OFDMA Systems and heterogeneous network scenario with femtocells is not considered. In $[27]$ a comprehensive survey on energy efficiency metrics is provided. Therefore, the reader is referred to this survey to access to the large number of papers related to energy efficiency.

The problem of power minimization in the OFDMA femtocell network has been rarely discussed in the literature. Minimizing the transmit power of FBS in downlink direction to reduce the interference level on the MUEs has been considered in $\underline{[28]}$ under co-channel deployment while satisfying the minimum requirements of FUEs. This work considers downlink Femtocell base station transmit power. No subchannel assignment was considered in their model and problems. Thus, this scenario is not applicable in practical cellular networks; however, it has a valuable framework and insight on the power minimization problems. Also, Lopez-Perez in $\underline{[11]}$ proposed a novel model for subchannel and power allocation in downlink to minimize the cell transmit power while taking realistic constraints into account. Although their proposed algorithms offer significant performance improvements in terms of user outages and cell capacity, these models are for downlink direction and uplink resource allocation is not addressed there. However the analysis of energy efficiency have attracted the attentions, in previous works, the problem of maximizing energy efficiency and minimizing power are not jointly considered for femtocell networks in uplink.

Different than the previous literature mentioned, in our study, we consider joint power and channel allocation for uplink for two different objectives: power minimization and energy efficiency maximization for a heterogeneous 
network scenario with overlay femtocell layer. For performance evaluation we have chosen to compare our algorithm with an existing algorithm $\underline{[29]}$ and equal power allocation algorithm as a benchmark.

\subsection{Contribution}

In this paper, to minimize the power consumption and maximize the energy efficiency of the heterogeneous networks with femtocells we have formulated two mixed-integer non-linear optimization problems. To satisfy the minimum required rate of users, different constraints are imposed. In order to see the effect of the high data rate users, two service classes are considered. The first class includes users demanding a high data rate and the second class includes the users with low data rate requirement. We also consider the co-channel spectrum allocation for better spectral reuse and femtocells operating in closed access mode. Furthermore, we consider a maximum acceptable interference level on each subchannel to preserve the QoS of MUEs. The main contributions of the paper are as follows:

- We have formulated the energy efficiency maximization and power minimization problems for the uplink of heterogeneous networks. Since the formulated problems for joint channel and power allocation are non-convex, the problems are converted into a convex form by using the time sharing concept. The optimal solutions for these problems are then obtained in closed-form by KarushKuhnTucker (KKT) conditions on the Lagrangian of the optimization problems.

- We have investigated the resource allocation problem in heterogeneous networks for given objectives. In order to perform the resource allocation in the system, we have proposed two iterative power and channel allocation algorithms. Moreover, a simplified algorithm is introduced to reduce the complexity of proposed algorithms. In the uplink power model, we have also considered the circuit power and its effects on the proposed algorithms. In addition, the complexity of the proposed algorithms has been analyzed.

- Finally, we have analyzed and compared the proposed resource allocation methods with energy efficiency criterion. It can be seen that significant performance improvement is attained in comparison with existing algorithms.

The rest of the paper is organized as follows. In Section 2, the system model and problem formulation is presented. Resource allocation strategies are proposed in Section 3 while three algorithms with their complexity analysis are presented in section 4 . To verify the performance of the proposed algorithms simulation results are presented in section 5 . The concluding remarks are shown in section 6 . 


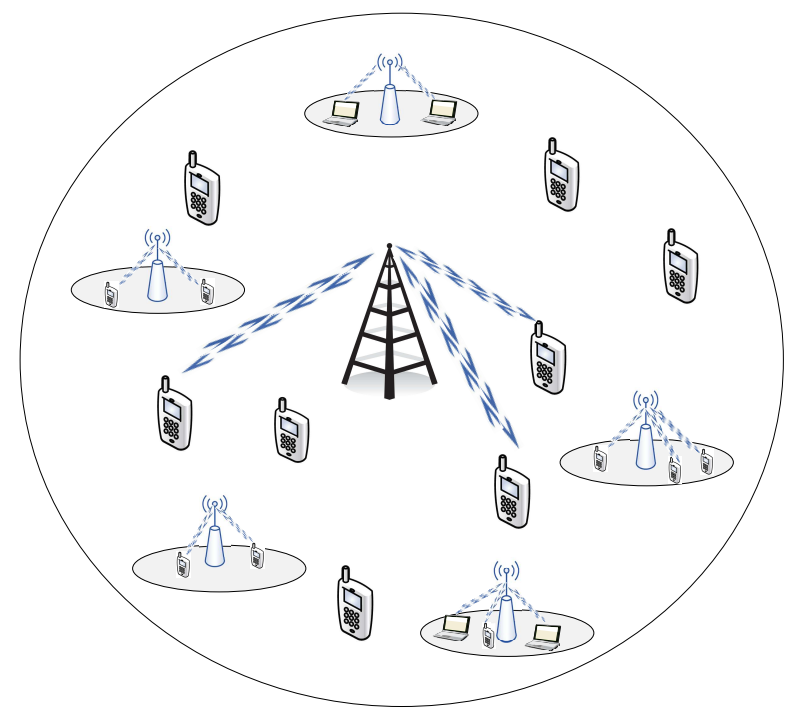

Fig. 1: System model of Two-tier femtocell network.

\section{System Model and Problem Formulation}

\subsection{System Model}

According to Fig.1, a two tier OFDMA cellular network is considered in this study. The system model parameters and descriptions are provided in Table 1. The FUEs and MUEs are distributed uniformly in the cell area. The total bandwidth is shared by the FUEs and MUEs in uplink direction. In other words, we consider the co-channel deployment network. Thus, it is imperative to limit the interference caused by FUEs on MBS that might have higher priority to use the bands in networks [14]. In addition, FUEs that are connected to the same FBS cannot share the same subchannel. We assumed closed access mode for all femtocells which means only those who are defined by the femtocell owner can connect to the femtocells $[30]$.

Generally, all FUEs demand different QoS in terms of their data rate. We consider two types of users corresponding to two different rate requirements. $|H D R|+|L D R|=F$ and $H D R_{k} \cap L D R_{K}=\varnothing$, where $|H D R|,|L D R|$ denotes the number of high data rate users and low data rate users. Our channel model is composed of three parts; large scale fading that is chosen based on [31], small scale fading and shadow fading. Let $P_{w, n}^{M}$ be the transmit power of MUE $w$ on subchannel $n$ and $P_{k, u, n}^{F}$ be the transmit power of FUE $u$ connected to FBS $k$ on subchannel $n$. Then, the received SINR of user $u$ at the FBS $k$ is calculated as:

$$
\gamma_{k, u, n}=\frac{p_{k, u, n}^{F} h_{k, u, n}^{F}}{p_{w, n}^{M} h_{k, w, n}^{F M}+\sigma^{2}}
$$


Table 1: System model parameter definitions and notations

\begin{tabular}{ll}
\hline Notation & Description \\
\hline$W$ & Bandwidth \\
$K$ & Number of channels \\
$M$ & Number of femtocells \\
$F$ & Number of active MUEs \\
$P_{c}$ & Number of users in each femtocell \\
$N_{0}$ & Circuit power \\
$\gamma_{k, u, n}$ & Noise power spectral density \\
$I_{t h}$ & Maximum of FUE $u$ in femtocell $k$ on subchannel $n$ \\
$R_{k, u}$ & Required rate by the $u$ th user in $k$ th femtocell \\
$a_{k, u, n}$ & Binary channel allocation variable of femtocell $k$ \\
$h_{k, w, n}^{F M}$ & in subchannel $n$ for user $u$ \\
$h_{k, u, n}^{M F}$ & Channel gain from MUE $w$ to femtocell $k$ in sub- \\
$h_{k, u, n}^{F}$ & Channel $n$ \\
& channel gain from FUE $u$ of femtocell $k$ in sub- \\
& Channel gain from FUE $u$ of femtocell $k$ in sub- \\
& chan to the FBS
\end{tabular}

The first term in the denominator of $\gamma_{k, u, n}$ is interference caused by MUEs on the subchannel $n$ on the FBS $k$ and the second term is thermal noise, which is denoted by $\sigma^{2}$. The interference caused by nearby FUEs of neighboring femtocells as well as other cells interferences on subchannel $n$ is absorbed in thermal noise. This assumption is realistic particularly in sparse deployed femtocells with a high penetration loss of residential buildings, where the interference level is negligible [29]. The spectral efficiency of each FUE in femtocell $k$ on subchannel $n$, which is measured in $b p s / \mathrm{Hz}$, follows the normalized Shannon rule which is defined as:

$$
C_{k, u, n}^{F}=\log _{2}\left(1+S I N R_{k, u, n}^{F}\right)
$$

The total energy efficiency of the system is defined as the ratio of the sum rate and the total power consumption of all users:

$$
E E^{T}=\frac{\sum_{k=1}^{K} \sum_{u=1}^{F} \sum_{n=1}^{N} a_{k, u, n} C_{k, u, n}^{F}}{\sum_{k=1}^{K} \sum_{u=1}^{F}\left(\sum_{n=1}^{N} a_{k, u, n} p_{k, u, n}+P_{c}\right)}
$$

where $P_{c}$ is a constant circuit power consumed at the UE representing the power consumption of device electronics while the transmit power is used for reliable data transmission [32]. As in [33], UE power model includes both the circuit power and the transmit power. 


\subsection{Problem Formulation}

In this subsection, we aim to optimize our system from two standpoints, efficiency and power. To achieve our goal, two main optimization problems are formulated. The first problem aims to minimize the aggregate power of all FUEs while the second problem focuses on maximizing the energy efficiency and they are referred to as problem I and problem II respectively.

Minimum Power Problem Formulation:

The optimization problem I can be formulated as:

$$
\begin{array}{ll}
\min _{a_{k, u, n}, p_{k, u, n}} \sum_{k=1}^{K} \sum_{u=1}^{F} \sum_{n=1}^{N} a_{k, u, n} p_{k, u, n} & \\
\text { s.t. } & \forall k, n \\
\sum_{u=1}^{F} a_{k, u, n} \leq 1 & \forall k, u, n \\
a_{k, u, n} \geq 0 & \forall k, u \\
\sum_{n=1}^{N} a_{k, u, n} p_{k, u, n} \leq P_{\max } . & \forall u, k \\
\sum_{n=1}^{N} a_{k, u, n} C_{k, u, n} \geq R_{k, u} & \\
\sum_{k=1}^{K} \sum_{u=1}^{F} a_{k, u, n} p_{k, u, n} h_{k, u, n}^{M F} \leq I_{n}^{t h} . & \forall n
\end{array}
$$

The objective function to be minimized in (4) is the total aggregate power consumed by all FUEs and $a_{k, u, n} \in\{0,1\}$ is a variable that indicates whether a subchannel $n$ is assigned to a user. In (4) we omit the circuit power because we have assumed that it is constant during the transmission and therefore it has no effect on the final solution of problem. Also, each subchannel must be dedicated to at most one FUE in each femtocell. Thus, constraints (5) and (6) are applied to the problem. The constraint shown in (7) is imposed to ensure that the total transmit power of each FUE does not exceed the maximum allowable value which is denoted by $P_{\max }$. The constraint shown in (7) does not affect the solution significantly due to the power minimization nature of the problem. However, the interference plays a key role in finding the final solution; (9) guarantees that the total interference of all FUEs on each subchannel is lower than the predefined threshold denoted by $I_{n}^{t h}$. Therefore, by satisfying (9), the transmitting power of FUEs does not disturb the MUEs QoS significantly. In addition, each user requires a data rate which needs to be satisfied by the constraint that is imposed in (8), where $C_{k, u, n}$ is defined in (2).

\section{Energy Efficiency Problem Formulation:}

The goal of this optimization problem is to maximize the total energy efficiency, $E E^{T}$, defined in (3) and the constraints are the same as the previous 
optimization problem, as in the following:

$$
\begin{aligned}
& \max _{a_{k, u, n}, p_{k, u, n}} E E^{T} \\
& \text { s.t. (5) to (9) }
\end{aligned}
$$

Energy efficiency is a metric which quantifies how efficiently resources are used. It is measured by bit/Hz/joule, which is the spectral efficiency over power consumption. In some sense, this is a multi objective problem that maximizes the throughput and minimizes power at the same time. Therefore, this problem makes effort to transmit data with maximum possible data rate with minimum power consumption, while satisfying the constraints.

\section{Subchannel Assignment And Power Allocation Strategy}

The optimization problems I and II are mixed-integer non-convex problems, therefore it is hard to find their optimal solution [34]. In this section, the time sharing concept is used to transform the mixed-integer non-convex optimization problem to a convex one. Then, the resulting convex optimization problem is solved using the Lagrangian dual decomposition method [35]. First, the optimization problem $\mathbf{I}$ is solved then to deal with optimization problem II, we transform it to an equivalent problem which is similar to the first problem in form. Hence, the solution to the second problem will be straightforward and only the final results are given as we will explain later.

\subsection{Minimizing the Aggregate Power}

In the problem formulation, constraints (5) and (6) cause model to become mixed integer non-convex because $a_{k, u, n}$ is a binary variable. To find the optimal solution of such a problem an exhaustive search algorithm like brute-force must be applied. These approaches have a high computational complexity [29]. By means of heuristic search methods like the genetic algorithm, near optimal solutions can be obtained as well; however, global optimality of the solutions cannot be guaranteed [36].

The time sharing concept has been widely used to transform these kind of problems to convex optimization problems in multi channel multi user systems $[29],[37]$ and is introduced by Wong in [35]. Then, in [36] the zero duality gap of multicarrier systems was proved once the time sharing condition was fulfilled. To apply this concept to the proposed problem, instead of dealing with $a_{k, u, n}$ as a binary variable, it is treated as a continuous variable in the range of $[0,1] . a_{k, u, n}$ can be seen as a time-share factor which shows the fraction of time that a subchannel is assigned to a user. After relaxing the $a_{k, u, n}$ to be a continuous variable, and changing the variables $a_{k, u, n} p_{k, u, n}$ into a variable $s_{k, u, n}$ the resulting optimization problem will be convex.

The goal function in (4) is concave due to negative semi-definite elements of the Hessian matrix with respect to $a_{k, u, n}$ and $s_{k, u, n}$. The feasible set of 
goal function is convex because the inequality constraints in (6) to (9) are convex [34]. Therefore, the optimization problem becomes a convex optimization problem that has a unique optimal solution. In other words, the local solutions are optimal, and can be obtained in polynomial time [34]. In this section we use the Lagrangian dual decomposition method to solve the convex optimization problem. First we formed the Lagrangian function and then we have decomposed the problem. The Lagrangian function can be formulated as:

$$
\begin{aligned}
& \mathscr{L}\left(\left\{a_{k, u, n}\right\},\left\{p_{k, u, n}\right\}, \lambda, \nu, \mu, \eta\right) \\
& =\sum_{k=1}^{K} \sum_{u=1}^{F} \sum_{n=1}^{N} a_{k, u, n} p_{k, u, n} \\
& +\sum_{k=1}^{K} \sum_{u=1}^{F} \lambda_{k, u}\left(\sum_{n=1}^{N} a_{k, u, n} p_{k, u, n}-P_{\max }\right) \\
& +\sum_{n=1}^{N} \mu_{n}\left(I_{n}^{t h}-\sum_{k=1}^{K} \sum_{u=1}^{F} a_{k, u, n} p_{k, u, n} h_{k, u, n}^{M F}\right) \\
& +\sum_{k=1}^{K} \sum_{n=1}^{N} \eta_{k, n}\left(1-\sum_{u=1}^{F} a_{k, u, n}\right) \\
& +\sum_{k=1}^{K} \sum_{u=1}^{F} \nu_{k, u}\left(\sum_{n=1}^{N} a_{k, u, n} C_{k, u, n}-R_{k, u}\right),
\end{aligned}
$$

where $\eta, \lambda, \nu, \mu$ are dual variable vectors for (5), (7), (8) and (9), respectively. The condition in 6 will be considered in KKT conditions later. Now the dual function is:

$$
\begin{aligned}
& g(\lambda, \nu, \mu, \eta))= \\
& \min _{\left\{a_{k, u, n}\right\},\left\{p_{k, u, n}\right\}} \mathscr{L}\left(\left\{a_{k, u, n}\right\},\left\{p_{k, u, n}\right\}, \lambda, \nu, \mu, \eta\right)
\end{aligned}
$$

Thus, the dual problem becomes:

$$
\begin{aligned}
& \min _{\lambda, \nu, \mu, \eta} g(\lambda, \nu, \mu, \eta) \\
& \text { s.t. } \quad \lambda, \nu, \mu, \eta \geq 0
\end{aligned}
$$

To solve the problem, the idea of decomposition is used in which the main problem is broken into several smaller problems. The sub-problems cannot be solved separately, since they are coupled to each other with the constraints. Thus, the overall problem must be solved iteratively. In our problem, each femtocell solves the sub-problem for each subchannel of its users. Consequently, the Lagrangian dual function in (12) is decomposed into a master problem and $K \times N$ subproblems. We define $\mathscr{L}_{k, n}$ as:

$$
\mathscr{L}_{k, n}\left(\left\{a_{k, u, n}\right\},\left\{p_{k, u, n}\right\}, \lambda, \nu, \mu, \eta\right)=
$$




$$
\begin{aligned}
& \sum_{u=1}^{F}\left[a_{k, u, n} p_{k, u, n}+\lambda_{k, u} a_{k, u, n} p_{k, u, n}+\eta_{k, n} a_{k, u, n}\right. \\
& \left.-\nu_{k, u} a_{k, u, n} C_{k, u, n}^{F}+\mu_{n} a_{k, u, n} p_{k, u, n} h_{k, u, n}^{M F}\right] .
\end{aligned}
$$

Hence, the Lagrangian function introduced in (11) is as follows:

$$
\begin{aligned}
& \mathscr{L}\left(\left\{a_{k, u, n}\right\},\left\{p_{k, u, n}\right\}, \lambda, \nu, \mu, \eta\right) \\
& =\sum_{k=1}^{K} \sum_{n=1}^{N} \mathscr{L}_{k, n}\left(\left\{a_{k, u, n}\right\},\left\{p_{k, u, n}\right\}, \lambda, \nu, \mu, \eta\right) \\
& +\sum_{n=1}^{N} \mu_{n} I_{n}^{t h}-\sum_{k=1}^{K} \sum_{u=1}^{F} \nu_{k, u} R_{k, u} \\
& +\sum_{k=1}^{K} \sum_{u=1}^{F} \lambda_{k, u} P_{\text {max }}+\sum_{k=1}^{K} \sum_{n=1}^{N} \eta_{k, n} .
\end{aligned}
$$

Owing to KKT conditions to find the optimal solution of the subproblems, we set the derivative of (14) with respect to $\left\{a_{k, u, n}\right\}$ and $\left\{p_{k, u, n}\right\}$ to zero as follows:

$$
\begin{gathered}
\frac{\partial \mathscr{L}_{k, n}}{\partial p_{k, u, n}}=0 \\
\frac{\partial \mathscr{L}_{k, n}}{\partial a_{k, u, n}}=0 .
\end{gathered}
$$

By considering (16), it can be shown that:

$$
p_{k, u, n}=\frac{\nu_{k, u}}{\ln (2)\left(1+\lambda_{k, u}+\mu_{n} h_{k, u, n}^{M F}\right)}-\frac{I_{k, u, n}}{h_{k, u, n}}
$$

Therefore, according to (18) the power assigned to the user becomes:

$$
\tilde{p}_{k, u, n}=\left(p_{k, u, n}\right)^{+}
$$

where $(z)^{+}$is $\max (z, 0)$. It is noteworthy to see that the dual variable $\nu_{k, u}$ is related to the required rate of users, so if some users do not demand any rates, their dual variable $\nu_{k, u}$ will be zero. This forces the first term in (18) to become zero and due to (19) the power allocated to the user is forced to be zero. According to this interesting result, all the users are assumed to require different data rates. Furthermore, this result is in line with power minimization objective since if a user does not need any rate, in order to reduce the extra power, the system forces the transmit power to be zero. Taking (17) into account, the decision criterion to find the proper channel is derived as follows:

$$
\begin{aligned}
& \frac{\partial \mathscr{L}_{k, n}}{\partial a_{k, u, n}}= \\
& p_{k, u, n}^{F}\left(1+\lambda_{k, u}+\mu_{n} h_{k, u, n}^{M F}\right)-\nu_{k, u} C_{k, u, n}^{F}+\eta_{k, n} .
\end{aligned}
$$


The last term in (20), which is calculated in MBS, is not related to the users so it will be sent to each femtocell by MBS. This term does not play any role in subchannel assignment, even though it has an effect on updating the variables. Finally, to assign the subchannels, the channel criterion $\left(C C_{k, u, n}\right)$ is introduced to make a decision based on that. $C C_{k, u, n}$ is similar to (20) except for the last term:

$$
\begin{gathered}
C C_{k, u, n}=\quad p_{k, u, n}^{F}\left(1+\lambda_{k, u}+\mu_{n} h_{k, u, n}^{M F}\right) \\
-\nu_{k, u} C_{k, u, n}^{F}
\end{gathered}
$$

Based on a channel criterion, $C C_{k, u, n}$, we introduce the following rule to assign a subchannel to a user. Each subchannel is allocated to a user with lower $C C_{k, u, n}$ in each femtocell. In addition, by using the time sharing concept and a decision criterion, $C C_{k, u, n}$, we relax a condition; therefore, we seek for a near optimal solution with semi distributed resource allocation strategy.

$$
\tilde{a}_{k, \tilde{u}, n}=\left.1\right|_{\tilde{u}=\min C C_{k, u, n}} \forall k, n
$$

By allocating a subchannel to a user, FUE must transmit power on that subchannel. Therefore, it is in contrast with the power minimization objective of this problem. As a result, the lowest increase in total power allocated to FUEs is more preferable. In other words, a channel with lower $C C_{k, u, n}$ regarding to (20) and (21) is superior to other channels. Another noteworthy result from considering (21) and (22) is that time sharing is ignored. In other words, while this problem is solved using time-sharing, this system can get better performance in terms of interference management. This result is in accordance with the results shown by Zhang in [29]. They suggested that improvement in the performance without time-sharing is due to preventing a co-tier interference in femtocells. An iterative approach is used to update the dual variables when applying the sub-gradient method as follows:

$$
\begin{aligned}
& \lambda_{k, u}^{(t+1)}= \\
& {\left[\lambda_{k, u}^{(t)}-\alpha_{1}^{(t)}\left(P_{\max }-\sum_{n=1}^{N} a_{k, u, n} p_{k, u, n}\right)\right]^{+}, \forall k, u} \\
& \nu_{k, u}^{(t+1)}= \\
& {\left[\nu_{k, u}^{(t)}-\alpha_{2}^{(t)}\left(\sum_{n=1}^{N} a_{k, u, n} C_{k, u, n}^{F}-R_{k, u}\right)\right]^{+}, \forall k, u} \\
& \mu_{n}^{(t+1)}= \\
& {\left[\mu_{n}^{(t)}-\alpha_{3}^{(t)}\left(I_{n}^{t h}-\sum_{k=1}^{K} \sum_{u=1}^{F} a_{k, u, n} p_{k, u, n} h_{k, u, n}^{M F}\right)\right]^{+}, \forall n}
\end{aligned}
$$

where $\alpha_{1}^{(t)}, \alpha_{2}^{(t)}, \alpha_{3}^{(t)}$ are step sizes and must be chosen carefully because they control the convergence of the algorithms. In addition, they have to follow 
some rules as:

$$
\begin{aligned}
& \lim _{T \rightarrow \infty} \sum_{t=1}^{T} \alpha_{i}^{(t)} \rightarrow \infty, \\
& \lim _{t \rightarrow \infty} \alpha_{i}^{(t)}=0,
\end{aligned}
$$

where $i \in\{1,2,3\}$ and $t \in\left\{1, \ldots, T_{\max }\right\}$ which $T_{\max }$ is the maximum number of iterations. In each femtocell, (24) and (25) are updated iteratively and all they need are provided by local information. In (21) the effect of $\eta$ is neglected, thus FBSs do not need extra information except their local data to perform channel assignment and power allocation. Also, (26) is updated in MBS so it needs information about the interference of the users who share the same subchannel. Information about interference can be obtained by channel gain estimation as well as users power information, which is provided through backhaul. Finally, $\eta$ will be sent to each FBS over the backhaul to update its parameters such as allocated power. Hence, by updating dual variables by (24), (25) and (26) power and subchannels are assigned dynamically and once they are converged, the ultimate solution is reported by the algorithm.

\subsection{Maximizing Energy Efficiency}

The optimization problem II is convex-concave fractional programming and the Dinkelbach algorithm $\underline{[38]}$ can be used to find its optimal solution $\underline{[26]}$. In order to solve problem II, it should be converted to a convex form. To simplify our notation, the nominator and denominator of (3) is redefined as $C^{T}$ and $P^{T}$, respectively. Then, the objective function is as follows:

$$
\xi=\frac{C^{T}}{P^{T}} \Rightarrow C^{T}-\xi P^{T}=0 .
$$

Therefore, the optimization problem II can be formulated as follows:

$$
\begin{array}{r}
\min _{a_{k, u, n}, p_{k, u, n}}-\left(C^{T}-\xi P^{T}\right) \\
\text { s.t. }(5) \text { to }(9)
\end{array}
$$

The fractional objective function is linearized in (29) and the problem formation is similar to the problem I. Consequently, the solution procedure is straightforward and is similar to that of the optimization problem $\mathbf{I}$ with some adjustments. Performing the same procedure as in optimization problem $\mathbf{I}$, the final results are provided. It is worth mentioning that $\xi$ must be calculated and updated in MBS, solving (28) for $\xi$ and the value must be sent to each FBS in each iteration. The channel criterion is:

$$
\begin{aligned}
& C C_{k, u, n}= \\
& p_{k, u, n}^{F}\left(\xi+\lambda_{k, u}+\mu_{n} h_{k, u, n}^{M F}\right)-\left(1-\nu_{k, u}\right) C_{k, u, n}^{F} .
\end{aligned}
$$


The power assigned to a user on each subchannel is:

$$
p_{k, u, n}=\frac{\left(1+\nu_{k, u}\right)}{\ln (2)\left(\xi+\lambda_{k, u}+\mu_{n} h_{k, u, n}^{M F}\right)}-\frac{I_{k, u, n}}{h_{k, u, n}}
$$

\section{Resource Allocation Algorithms and Complexity Analysis}

Three algorithms are proposed to implement the procedure of power and subchannel allocation to the users while optimizing the objective function, meanwhile handling the constraints. In (18), (19), and (22) the equations to find optimal subchannel and allocated power for each user are derived but the procedure of assigning resources is still a question. In order to answer this, three algorithms are proposed. These methods are suitable to be used in FBS, because they both converge fast enough to be used in practical systems and need very low information to be exchanged with MBS. Furthermore, a low complex and distributed interference management scheme, called REFIM is proposed in $[39]$ to deal with feedback overhead over backhaul.

\subsection{Near Optimal Algorithms}

Algorithm 1 and 2 are presented to demonstrate the steps of the proposed resource allocation methods as will be discussed later.

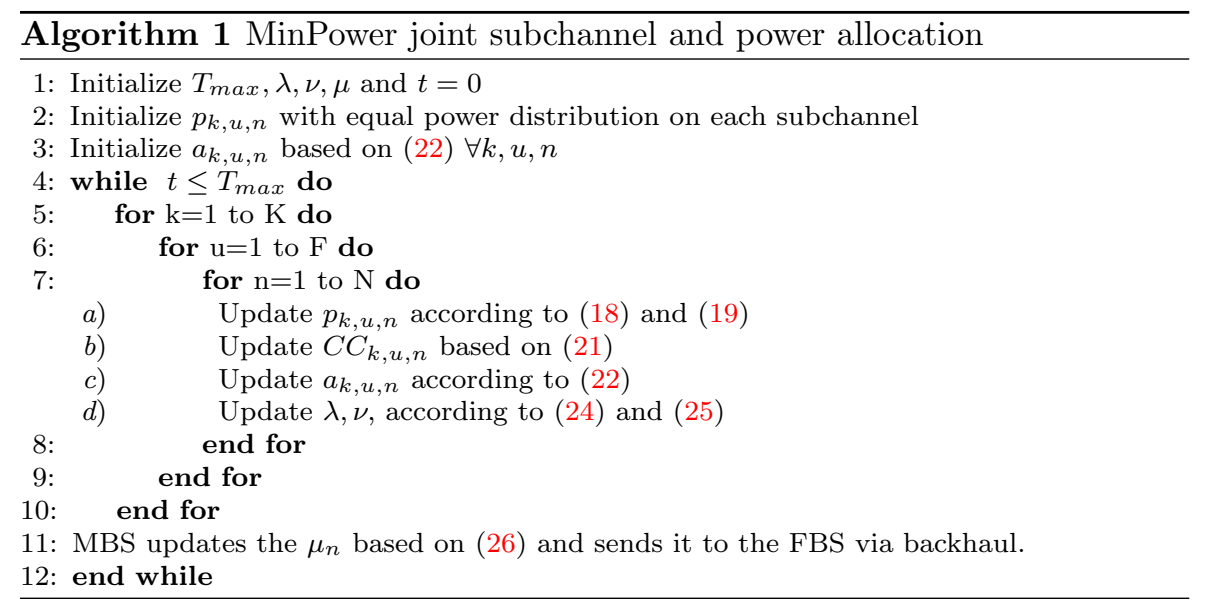

In Algorithm 1 and 2, for each femtocell all the parameters are available based on local data except $\mu_{n}$ and the channel gain $h_{k, u, n}^{M F}$. These parameters are required to compute the allocated power and channel criterion defined in (18), (31), and (21). The channel gain can be measured by FBS locally or MBS could approximate and send it to the FBS via backhaul. The complexity analysis of both algorithms are discussed later. 


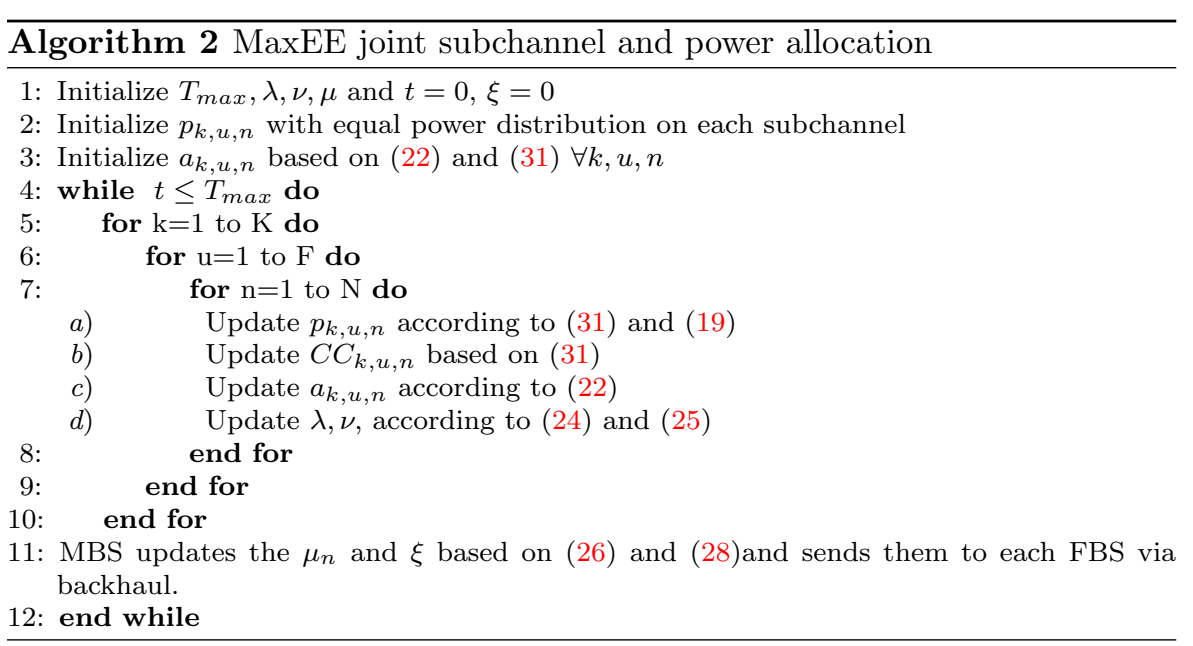

\subsection{Sub Optimal Algorithm}

The third proposed method is the simplified version of the first and second algorithms so that it becomes more practical to be used by femtocells. The simplified algorithm sacrifices the optimal solution to obtain a lower order of complexity. The complexity analysis of the proposed algorithms will be considered later. The simplified version of algorithm 1 and algorithm 2 contains two main steps: Subchannel allocation and power allocation, as introduced in algorithm 3. Algorithm 3 is more preferable due to its simple implementation; however, it is not able to find the near optimal solutions. The third or simplified algorithm consists of two major parts. The first part is devoted to channel allocation that allocates a suitable subchannel to the FUEs and the second part is about power updating of each user on each subchannel, that is, FBS adjusts FUE's transmit power to minimize the aggregate power consumed by FUEs. In other words, in the former part we make sure that by uniform power allocation, all the users will reach their required rate meanwhile, in the latter no channel allocation will be performed and only the power will be updated, hence the complexity will be reduced which will be addressed in the next section.

\subsection{Complexity Analysis}

Complexity analysis plays an important role in comparing the time efficiency of different algorithms. In this subsection, the complexity order of the proposed algorithms is discussed. Generally, Algorithm 1 and 2 offer similar complexity because they are almost similar in procedure. Therefore, we focus on the complexity order of 1 . In Algorithm 1, during each iteration, calculating (21) and updating (22) need $K F N$ operations, independently. Furthermore, $K F$ calculations are required for updating the dual variables $\lambda$ and $\nu$ according 


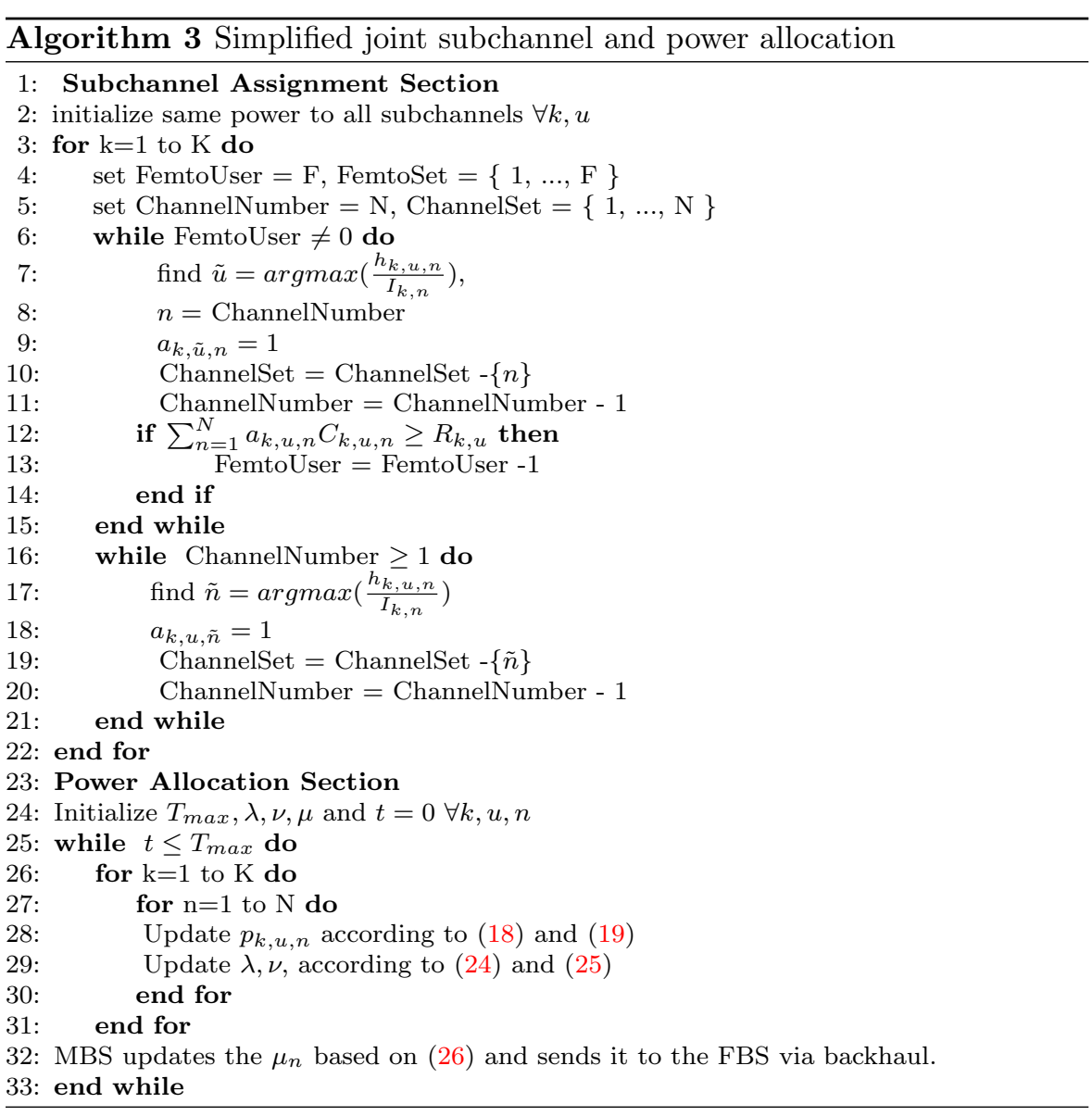

to (24) and (25), respectively. The parameter $\mu$ is also calculated in MBS for each subchannel and its complexity order is $\mathrm{O}(\mathrm{N})$ per iteration. Let algorithm 1 converge after $T_{\text {conv }}$ iterations, therefore, its total complexity becomes $O\left(T_{\text {conv }}(K F N)^{2}\right)$. Although all $K, F$ and $N$ are fixed network parameters, $T_{\text {conv }}$ is not a constant predefined value. The results of our preliminary tests show that smart initialization of parameters such as dual variables and step sizes can provide a smaller $T_{\text {conv }}$. It should be noted that updating the dual variables after the execution of the iteration loop on the subchannels can reduce the complexity of algorithm 1 . Furthermore, it is possible to update $a_{k, u, n}$ for all the users of the same femtocell at the same time.These modifications in the procedures not only reduce the complexity order, but also result in reducing the total execution time of the algorithm, which is critically important, even though it requires more memory.

Algorithm 3 suggests a simplified procedure to solve the proposed optimization problem. Finding a proper user in each subchannel for every femtocell requires total $K N F$ operations while updating $\mu$ for each subchannel 
calls for total $K N^{2}$ calculations. Therefore, the total complexity order of the subchannel allocation section is $O(K N(F+N))$. The complexity analysis of the second part of algorithm 3 is almost similar to what is done in algorithm 1. The loop on the number of femtocells is reduced and subsequently, the overall required calculation is $O\left(K^{2} N^{2} F T_{\max }\right)$. To further reduce the complexity, it is also possible to update the dual variables for each femtocell instead of all of them, therefore the complexity becomes $O\left(K N^{2} F T_{\max }\right)$. To sum up the analysis here, the total complexity order of algorithm 3 is $O\left(K N^{2} F T_{\max }\right)$. Femtocells can use both algorithms but the proposed simplified scheme provides lower complexity than algorithm 1 and algorithm 2; however, in return it sacrifices the optimality of the final solution.

To compare the complexity order of the proposed algorithms with existing schemes, we consider the exhaustive search and genetic algorithm as two alternative approaches towards solving the optimization problem. $O\left(K F^{N}\right)$ is a complexity order of exhaustive search whereas, NSGA II, as an example of the genetic algorithm, has a complexity order of $O((N p G)(F K N+O b N p))$ where $G$ is the number of generations, $N_{p}$ is the population number and $O_{b}$ is the number of objectives which is 1 in our case [19]. The proposed algorithms have much lower complexity than NSGA II and exhaustive search knowing the fact that generally the number of generations and populations is high in NSGA II in comparison with $F, K, N$ [19].

\section{Simulation Results}

In the previous sections, three resource allocation methods are proposed aiming at total user power minimization and energy efficiency maximization. In this section the performance of the proposed algorithms is investigated. The scenario as depicted in Fig.1, is co-channel deployed femtocells that are overlaid on a macrocell. The simulation parameters and their corresponding values are summarized in Table 2. To make the system more realistic, we assume that each femtocell can serve up to $F$ users and they require two types of services with high and low QoS. The carrier frequency is set to $2 \mathrm{GHz}$ and thermal noise is considered as a zero mean Gaussian random variable with variance of $\sigma^{2}$ and power spectral density of $N_{0}=-174 \mathrm{dbm} / \mathrm{Hz}$, so $\sigma^{2}=(W / N) N_{0}$. Channel fading is composed of path loss, shadow fading and Rayleigh fading. Pathloss models are chosen based on [31], shadow fading is modeled as zero mean log normal distributions with variance of $10 d b$ and $8 d b$ for MUEs and FUEs, respectively. Besides, Rayleigh fading channel gains are modeled as a unit-mean exponential distribution. Femtocells have a coverage radius of $20 \mathrm{~m}$, furthermore, they are distributed uniformly in a macrocell coverage with radius of $1000 \mathrm{~m}$. In our simulations, femtocell centers have a distance, at least three times of their radius to that of the others; therefore femtocells coverage cannot overlap each other. This assumption is in line with the ignorance of the co-tier interference. 
Table 2: Simulation parameters values

\begin{tabular}{lll}
\hline Definition & Notation & Value \\
\hline Bandwidth & $W$ & $20 \mathrm{MHz}$ \\
Number of channels & $N$ & 50 \\
Number of active MUEs & $M$ & 50 \\
Number of femtocells & $K$ & 30 \\
Number of users in each femtocells & $F$ & 4 \\
Circuit power & $P_{c}$ & $0,100 \mathrm{~mW}$ \\
Maximum allowable interference & $I_{t h}$ & $-101 \mathrm{dbm}$ \\
Maximum transmit power of users & $P_{\max }$ & $23 \mathrm{dbm}$ \\
Noise power spectral density & $N_{0}$ & $-174 \mathrm{dbm} / \mathrm{Hz}$ \\
High quality service requirements & $R_{H D R}$ & $60 \mathrm{bs} / \mathrm{Hz}$ \\
Low quality service requirements & $R_{L D R}$ & $20 \mathrm{bps} / \mathrm{Hz}$
\end{tabular}

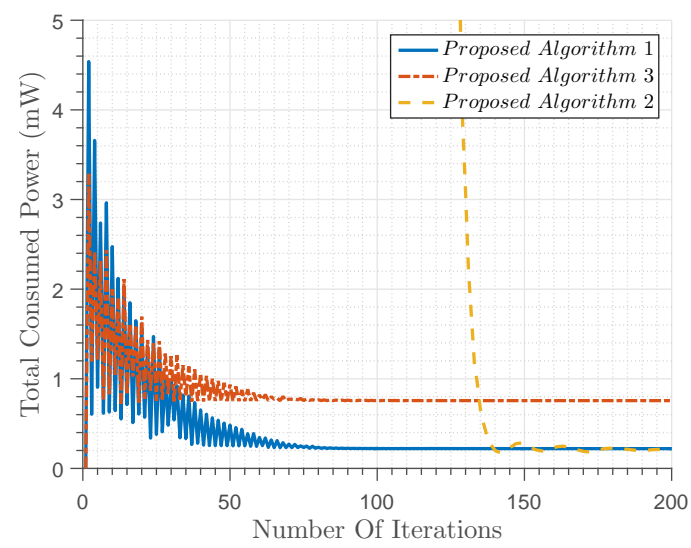

Fig. 2: Convergence performance of aggregate power consumption for proposed algorithms.

In Fig.2 and Fig.3, the convergence performance of the goal function for all proposed methods are depicted in which the number of femtocells and users in each femtocell are set to 30 and 6, respectively. Step sizes in updating the dual variables in (24),(25) and (26) have effects on the convergence rate of algorithms. By using the step size coefficient updating function $t^{-t . p}$ in our simulations, we let the early steps have greater effects on updating the variables. It can be seen that all algorithms converge rapidly proportional to the total number of users; therefore they are all suitable to be utilized in practical resource management in small cell scenarios. Also it can be seen from Fig. 2 and Fig.3 that the first algorithm outperforms the others in terms of power consumption and the second one outperforms the others in terms of energy efficiency. When it comes to the convergence performance, the third algorithm performs better in total power consumption convergence and the second algorithm performs worse because its objective function is not power. 


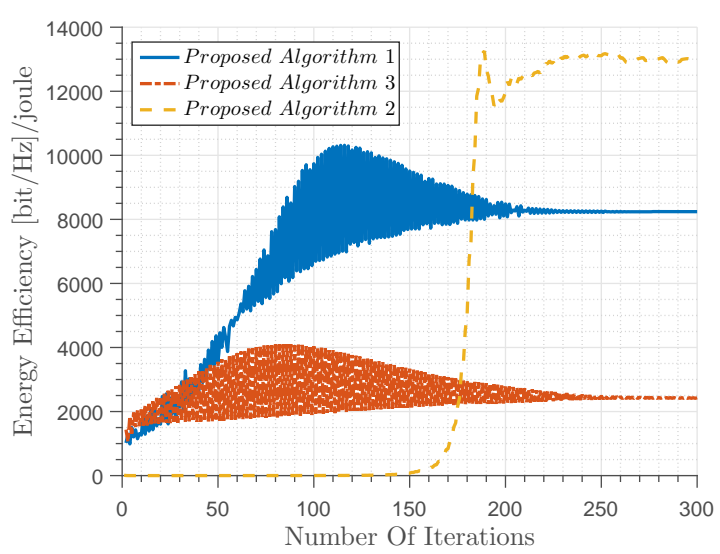

Fig. 3: Convergence performance of total system efficiency for proposed algorithms.

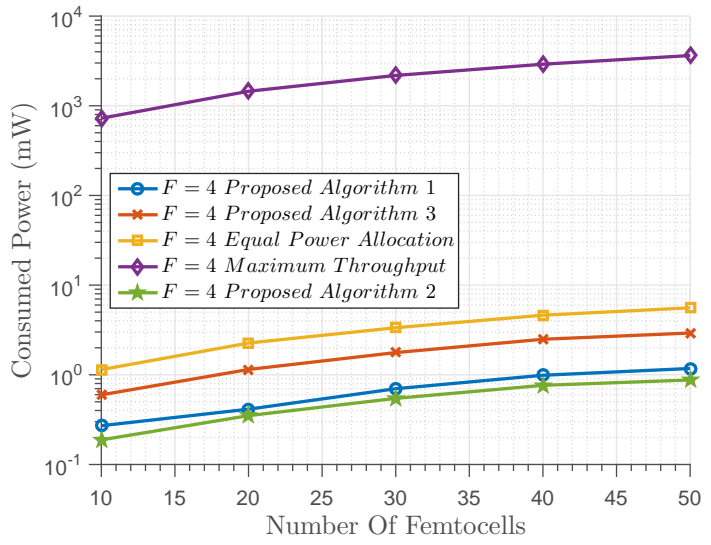

Fig. 4: Aggregate transmit power consumption Vs. number of femtocells

The total power consumption of the proposed algorithms for $F=4$ is demonstrated in Fig.4 for different femtocell numbers. As a reference and benchmark, we compare the power consumption of our algorithms with an existing algorithm [29], referred to as maximum throughput algorithm and equal power allocation. Equal power allocation is of industry interest because it is simple and easy to implement. In equal power allocation, we set the value of power to which the worst user reaches its required rate. As depicted in Fig.4, significant energy saving is attained in comparison with the existing solution and equal power allocation. All of our algorithms perform better than existing ones and also it can be seen that algorithm 1 and algorithm 2 outperform the simplified algorithm. Furthermore, in the proposed algorithms, when $F$ is set to 4 the aggregate power consumption does not increase dramatically 


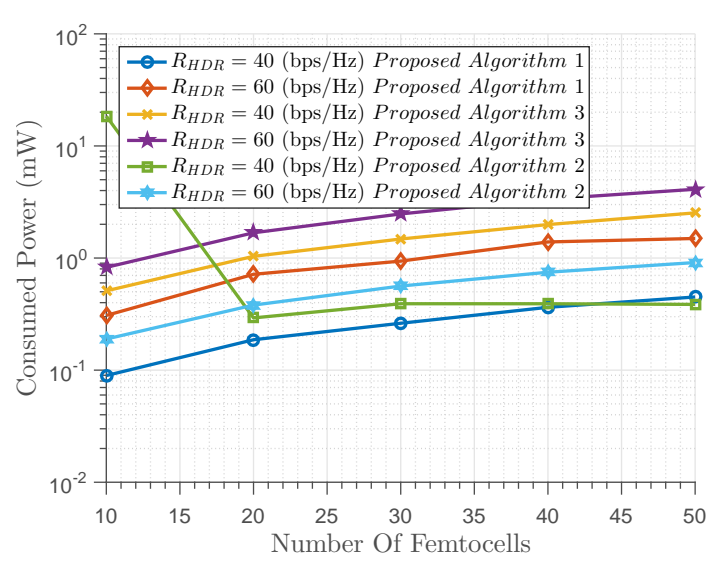

Fig. 5: Total transmit power consumption Vs. number of femtocells

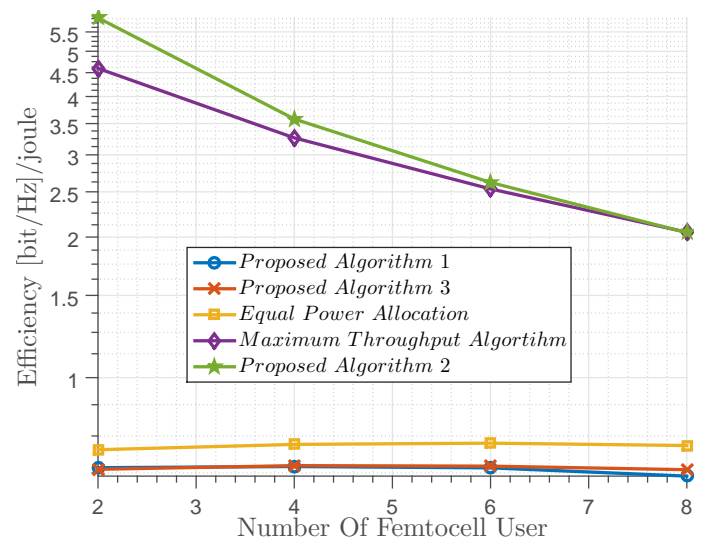

Fig. 6: Load impact effect on energy efficiency $P_{c}=100 \mathrm{~mW}$

since there is enough channel to be allocated to the users in each femtocell. Consequently, users do not need to use more power to reach their required rate. Generally, the simplified algorithm uses more power than algorithm 1 and 2 especially when the number of users in a cell is high e.g. $F=8$. This higher power consumption is due to the existence of more users and also the lower number of allocated subchannels to each user, which makes them use more power to reach their required rate. Although consumption power in the simplified algorithm is higher than algorithm 1 and algorithm 2, it is yet acceptable to be utilized in practical systems.

Total power consumption in terms of number of femtocells for different required rate of $H D R$ users is depicted in Fig.5. In this simulation, the required rate of $L D R$ users, $H D R$ users and number of users in each femtocell is set to $R_{L D R}=10 \mathrm{bps} / \mathrm{Hz}, R_{H D R}=40$ and $60 \mathrm{bps} / \mathrm{Hz}$ and $F=4$, respectively. 


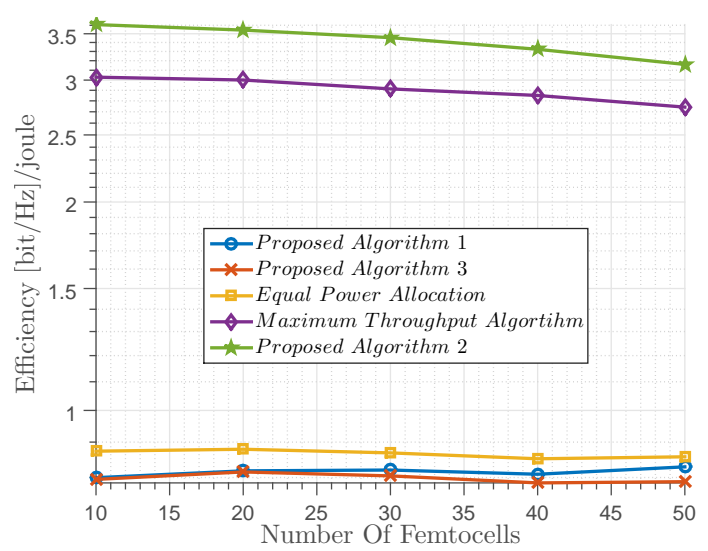

Fig. 7: Total Energy efficiency of proposed algorithms Vs. Number of femtocells $P_{c}=100 \mathrm{~mW}$

Interestingly, when the number of femtocells and the rate of high data rate users are set to $F=10$ and $R_{H D R}=40 \mathrm{bps} / \mathrm{Hz}$ the consumed power is relatively high in algorithm 2 . The reason is that when the number of femtocells and required rate of users are low the interference level is not a critical issue and the system has the freedom to allocate as much as power and channel to the users to maximize the energy efficiency. Furthermore, it is expected that demanding a greater data rate results in more power consumption, verified by Fig.5. Algorithm 1 outperforms the others with a gentle slope compared to other methods where the power consumption slope is relatively steep.

In Fig.6 the load impact effect on energy efficiency is investigated. It can be seen that the more user in femtocell is active the lower energy efficiency is achieved. This is because when there are more users, number of channels are limited accordingly, therefore each user must transmit more power on each channel and consequently the energy efficiency decreases.

The total energy efficiency of the system for different number of femtocells is demonstrated in Fig. 7 and Fig. 8. It can be seen that in Fig. 7 the best performance is for algorithm 2 while maximum throughput algorithm is the second best algorithm. When considering the $(3), P_{c}$ plays a critical role. However $P_{c}$ is circuit power and is not considered in transmission power, it has an effect on the energy efficiency from user's side. The circuit power dominates the transmit power, when minimizing the power, in the denominator of (3) because the transmission power is much lower than circuit power when minimizing the power. That is why the maximum throughput algorithm outperforms the algorithm 1 and 3. From the network perspective, it is valuable to ignore the $P_{c}$ and investigate the algorithms performance by considering only the transmission power. In Fig. 7 this issue is addressed. It can be seen that algorithm 2 represents the best performance while algorithm 1 and algorithm 3 still perform better than maximum throughput and the equal power algorithm. Due to the 


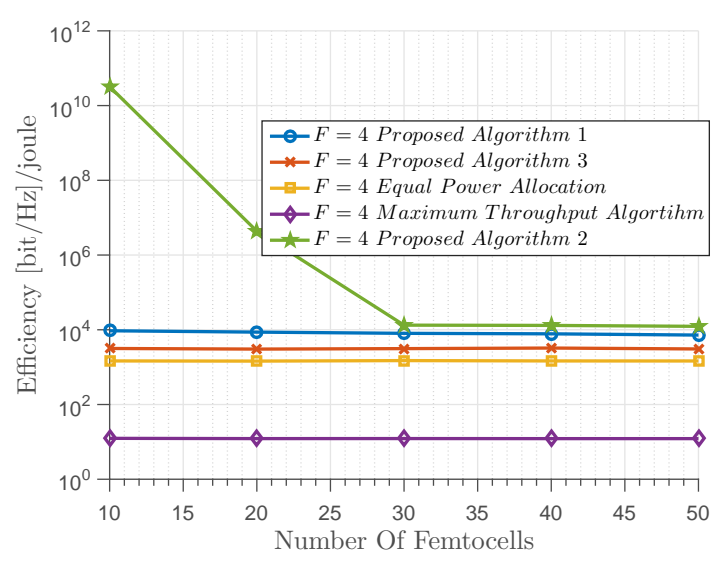

Fig. 8: Total Energy efficiency of proposed algorithms Vs. Number of femtocells $P_{c}=0$

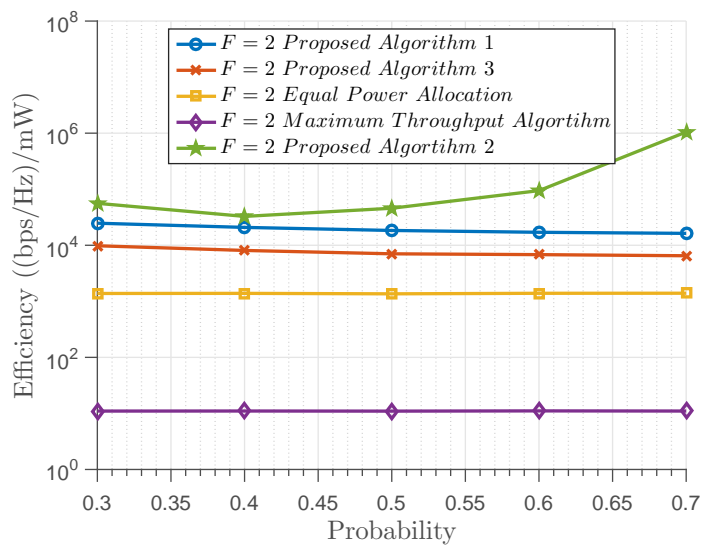

Fig. 9: Total Energy efficiency of proposed algorithms Vs. probability of being high data rate FUE $P_{c}=0$

high consumed power of the maximum throughput algorithm, it also fails to provide high energy efficiency. Thus, it is not an interesting algorithm in terms of efficiency, however it maximizes the throughput. Furthermore, the efficiency of all algorithms except algorithm 2 does not change dramatically with variation of femtocell numbers. There are two reasons for this phenomenon. Firstly, we ignore the co-tier interference; therefore the lower interference level is experienced in our simulations while in the dense scenario it might be crucial. In other words, if we consider the co-tier interference in our model, a steep downward trend is expected here. Secondly, all algorithms except algorithm 2 are far lower than their optimum efficiency level and their level of interference are relatively low and as a result by increasing the number of femtocells they 


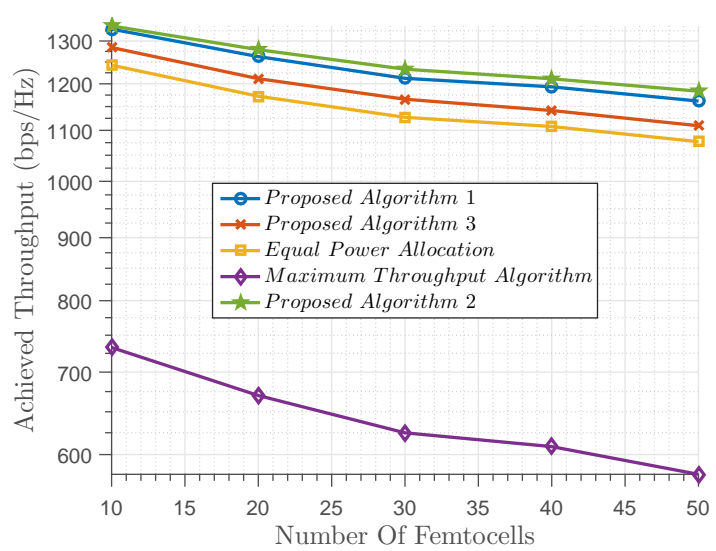

Fig. 10: The effect of proposed algorithms on MUE's rate

can still use their proper channel so their efficiency does not drop sharply. In contrast to other algorithms, algorithm 2 experiences a sharp decline. This is because of constraints on power and interference level on each channel. By increasing the number of femtocells there exist more users who are using the same channel. Consequently, the cross-tier interference, which is more severe with the higher number of femtocells, make users use their non optimal channel with more power in order to satisfy the interference constraints on all channels. Hence, their efficiency level becomes lower. Interestingly, with the higher number of femtocells, e.g. $K=40$ the difference between the efficiency level of algorithm 2 and others become lower and lower but it is still an upper bound.

In Fig.9, the effect of $H D R$ user probability in our system is depicted. The more the number of $H D R$ users are, the less efficiency we experience because high data rate users need more resources and considering these users costs the system. These users use more resources and the amount of available resources decrease so this results in lower efficiency.

To preserve the MUEs QoS, the maximum allowable threshold level is imposed on each channel but it is noteworthy to see the effect of algorithms on the MUEs rate. In Fig.10 this issue is explored. It can be seen that our proposed algorithms degrade the MUEs rate by 10 per cent while max throughput algorithm caused about 21 percent degradation in MUEs rate which means that our algorithms performed two times better than existing algorithms. Moreover, the total throughput of MUEs in our algorithms are twice to that of proposed algorithms. Interestingly, algorithm 2 outperform the others in terms of lower impact on MUEs QoS degradation although the degradation slope of the proposed algorithms are almost similar to each other.

In algorithm 3 channel assignment is done just once and then only power allocation is performed, while in algorithm 1 and algorithm 2 power allocation and channel assignment is performed simultaneously. Normalized deviation ratio (DR) of each user with regards to its required rate is defined as $D R_{k, u}=$ 


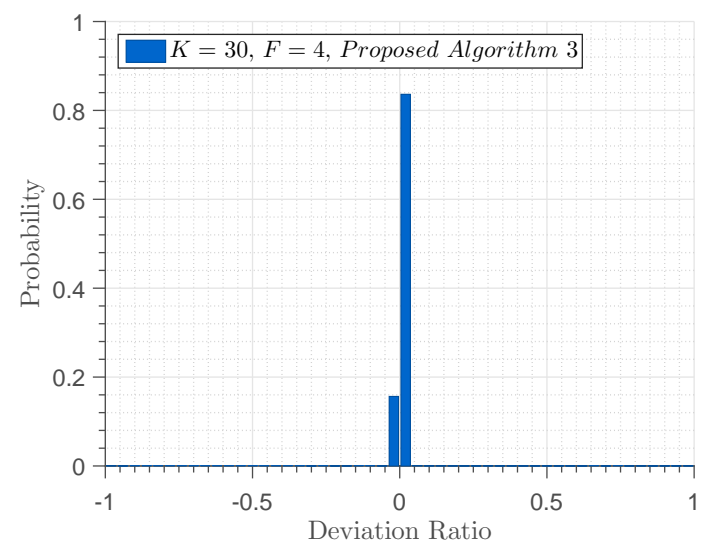

Fig. 11: Deviation ratio of algorithm 3.

$\frac{R_{k, u}^{a}}{R_{k, u}}-1$, where the achieved rate by user $u$ in femtocell $k$ is denoted by $R_{k, u}^{a}$. Fig.11 presents the histogram of $D R$ for the algorithm 3. The Number of users in each femtocell and number of femtocells are set to $F=4$ and $K=30$ in this simulation, respectively. According to Fig.11, in algorithm 3, 95\% of the users reach $95 \%$ of their required rate. Therefore, algorithm 3 behaves perfectly and almost all users reach their predefined rate. By increasing $F$, the system may become infeasible and thus the total number of users that do not reach their rate increases.

\section{Conclusion}

In this paper, two critical aspects of a cellular networks namely power and energy efficiency are considered. To utilize the available resources efficiently and to optimize the system with the given goal functions, two optimization problems were solved. First problem was dedicated to minimize the aggregate power of all FUEs and the second problem was devoted to maximize the energy efficiency of the system. In order to protect the QoS of macrocell users, a maximum allowable interference level was imposed on each subchannel and to guarantee the QoS of FUEs, the minimum required rate of them are satisfied. Knowing the inherent non-convexity of our primary problems, we applied the time-sharing concept to transform the non-convex problem to a convex one. Accordingly, the problems were decomposed to sub problems and were solved by the sub-gradient method. In addition, three resource allocation schemes were proposed to examine the problems. The first and second algorithm were proposed with a near optimal solution while they needed only little local information to perform the resource allocation distributively and limited data to exchange with MBS. Furthermore, to decrease the complexity order, we proposed a simplified algorithm, which satisfies the minimum QoS 
of the users.Finally, simulation results demonstrated significant enhancement in terms of energy efficiency and the proposed algorithms could achieve significant power savings, while the first and second algorithm outperformed the simplified algorithm. To extend this work joint resource allocation of MUEs and FUEs will be considered while some subchannels are exclusively dedicated to MUEs.

\section{References}

1. G. Mansfield, "Femtocells in the us market-business drivers and consumer propositions," FemtoCells Europe, pp. 1927-1948, 2008.

2. Y. Sun, R. P. Jover, and X. Wang, "Uplink interference mitigation for ofdma femtocell networks," Wireless Communications, IEEE Transactions on, vol. 11, no. 2, pp. 614625,2012

3. J. Tang, D. K. So, E. Alsusa, K. A. Hamdi, and A. Shojaeifard, "Resource allocation for energy efficiency optimization in heterogeneous networks," IEEE Journal on Selected Areas in Communications, vol. 33, no. 10, pp. 2104-2117, 2015.

4. Y. Lee, T. Chuah, K. Loo, and A. Vinel, "Recent advances in radio resource management for heterogeneous lte/lte-a networks," 2014.

5. P. Xia, V. Chandrasekhar, and J. G. Andrews, "Open vs. closed access femtocells in the uplink," Wireless Communications, IEEE Transactions on, vol. 9, no. 12, pp. 37983809, 2010.

6. K. Ahuja, Y. Xiao, and M. van der Schaar, "Efficient interference management policies for femtocell networks," Wireless Communications, IEEE Transactions on, vol. PP, no. 99, pp. 1-1, 2015.

7. S. M. A. El-atty and Z. M. Gharsseldien, "Performance analysis of an advanced heterogeneous mobile network architecture with multiple small cell layers," Wireless Networks, pp. 1-22, 2016.

8. Y. L. Lee, J. Loo, T. C. Chuah, and A. El-Saleh, "Fair resource allocation with interference mitigation and resource reuse in lte/lte-a femtocell networks," 2015.

9. T. Mao, G. Feng, L. Liang, S. Qin, and B. Wu, "Distributed energy-efficient power control for macro-femto networks," Vehicular Technology, IEEE Transactions on, vol. PP, no. 99, pp. 1-1, 2015.

10. A. Abdelnasser, E. Hossain, and D. I. Kim, "Clustering and resource allocation for dense femtocells in a two-tier cellular ofdma network," Wireless Communications, IEEE Transactions on, vol. 13, no. 3, pp. 1628-1641, 2014.

11. D. López-Pérez, X. Chu, A. V. Vasilakos, and H. Claussen, "Power minimization based resource allocation for interference mitigation in ofdma femtocell networks," Selected Areas in Communications, IEEE Journal on, vol. 32, no. 2, pp. 333-344, 2014.

12. T. Han, G. Mao, Q. Li, L. Wang, and J. Zhang, "Interference minimization in $5 \mathrm{~g}$ heterogeneous networks," Mobile Networks and Applications, vol. 20, no. 6, pp. 756-762, 2015.

13. L. B. Le, D. Niyato, E. Hossain, D. I. Kim, and D. T. Hoang, "Qos-aware and energyefficient resource management in ofdma femtocells," Wireless Communications, IEEE Transactions on, vol. 12, no. 1, pp. 180-194, 2013.

14. D. T. Ngo, S. Khakurel, and T. Le-Ngoc, "Joint subchannel assignment and power allocation for ofdma femtocell networks," Wireless Communications, IEEE Transactions on, vol. 13, no. 1, pp. 342-355, 2014.

15. A. Alitaleshi, R. Ghazizadeh, and H. Kalbkhani, "Ameliorated resource allocation in two-tier femtocell-macrocell networks with six directional antennas for macrocells," Wireless Personal Communications, vol. 86, no. 3, pp. 1493-1508, 2016. [Online]. Available: http://dx.doi.org/10.1007/s11277-015-3002-1

16. A. Shahid, S. Aslam, H. S. Kim, and K.-G. Lee, "Distributed joint resource and power allocation in self-organized femtocell networks: A potential game approach," Journal of Network and Computer Applications, vol. 46, pp. 280-292, 2014. 
17. X. Ge, T. Han, Y. Zhang, G. Mao, C.-X. Wang, J. Zhang, B. Yang, and S. Pan, "Spectrum and energy efficiency evaluation of two-tier femtocell networks with partially open channels," Vehicular Technology, IEEE Transactions on, vol. 63, no. 3, pp. 1306-1319, 2014.

18. H. B. Jung and D. K. Kim, "Power control of femtocells based on max-min fairness in heterogeneous networks," Communications Letters, IEEE, vol. 17, no. 7, pp. 1372-1375, 2013.

19. N. Sharma, D. Badheka, and A. Anpalagan, "Multiobjective subchannel and power allocation in interference-limited two-tier ofdma femtocell networks," Systems Journal, IEEE, vol. PP, no. 99, pp. 1-12, 2014.

20. S. Gheitanchi, F. Ali, and E. Stipidis, "Particle swarm optimization for adaptive resource allocation in communication networks," EURASIP Journal on Wireless Communications and Networking, vol. 2010, p. 2, 2010.

21. H. Wang and Z. Ding, "Power control and resource allocation for outage balancing in femtocell networks," Wireless Communications, IEEE Transactions on, vol. 14, no. 4, pp. 2043-2057, 2015.

22. H. Zhang, C. Jiang, N. Beaulieu, X. Chu, X. Wang, and T. Quek, "Resource allocation for cognitive small cell networks: A cooperative bargaining game theoretic approach," Wireless Communications, IEEE Transactions on, vol. PP, no. 99, pp. 1-1, 2015.

23. F. Haider, C.-X. Wang, B. Ai, H. Haas, and E. Hepsaydir, "Spectral/energy efficiency tradeoff of cellular systems with mobile femtocell deployment," IEEE Transactions on Vehicular Technology, vol. 65, no. 5, pp. 3389-3400, 2016.

24. C. An, R. Xie, H. Ji, and Y. Li, "Pricing and power control for energy-efficient radio resource management in cognitive femtocell networks," International Journal of Communication Systems, vol. 28, no. 4, pp. 743-761, 2015.

25. G. Miao, N. Himayat, G. Y. Li, and S. Talwar, "Distributed interference-aware energy-efficient power optimization," Wireless Communications, IEEE Transactions on, vol. 10, no. 4, pp. 1323-1333, 2011.

26. L. Xu, G. Yu, and Y. Jiang, "Energy-efficient resource allocation in single-cell ofdma systems: Multi-objective approach," 2015.

27. Y. Li, H. Celebi, M. Daneshmand, C. Wang, and W. Zhao, "Energy-efficient femtocell networks: challenges and opportunities," Wireless Communications, IEEE, vol. 20 no. 6, pp. 99-105, 2013.

28. M. R. Mili and K. A. Hamdi, "On the minimum transmit power in cochannel femtocells," Communications Letters, IEEE, vol. 16, no. 7, pp. 1026-1029, 2012.

29. H. Zhang, C. Jiang, N. Beaulieu, X. Chu, X. Wen, and M. Tao, "Resource allocation in spectrum-sharing ofdma femtocells with heterogeneous services," Communications, IEEE Transactions on, vol. 62, no. 7, pp. 2366-2377, July 2014.

30. G. De La Roche, A. Valcarce, D. López-Pérez, and J. Zhang, "Access control mechanisms for femtocells," Communications Magazine, IEEE, vol. 48, no. 1, pp. 33-39, 2010.

31. E. U. T. R. Access, "Further advance-ments for e-utra physical layer aspects," 3GPP TR 36.814, Tech. Rep., 2010.

32. G. Miao, N. Himayat, G. Y. Li, and S. Talwar, "Low-complexity energy-efficient scheduling for uplink ofdma," IEEE Transactions on Communications, vol. 60, no. 1, pp. 112$120,2012$.

33. G. Miao, N. Himayat, and G. Y. Li, "Energy-efficient link adaptation in frequencyselective channels," IEEE Transactions on Communications, vol. 58, no. 2, pp. 545-554, 2010.

34. S. Boyd and L. Vandenberghe, Convex optimization. Cambridge university press, 2004.

35. C. Y. Wong, R. S. Cheng, K. B. Lataief, and R. D. Murch, "Multiuser ofdm with adaptive subcarrier, bit, and power allocation," Selected Areas in Communications, IEEE Journal on, vol. 17, no. 10, pp. 1747-1758, 1999.

36. W. Yu and R. Lui, "Dual methods for nonconvex spectrum optimization of multicarrier systems," Communications, IEEE Transactions on, vol. 54, no. 7, pp. 1310-1322, 2006.

37. J.-H. Yun and K. G. Shin, "Adaptive interference management of ofdma femtocells for co-channel deployment," Selected Areas in Communications, IEEE Journal on, vol. 29 no. 6, pp. 1225-1241, 2011.

38. W. Dinkelbach, "On nonlinear fractional programming," Management Science, vol. 13 , no. 7, pp. 492-498, 1967. 
39. K. Son, S. Lee, Y. Yi, and S. Chong, "Refim: A practical interference management in heterogeneous wireless access networks," IEEE Journal on Selected Areas in Communications, vol. 29, no. 6, pp. 1260-1272, June 2011. 